\title{
Ruolo di Helicobacter pylori nella patologia gastroenterica dei pazienti emodializzati
}

\author{
V. Barbera ${ }^{1}$, E. del Giudice ${ }^{1}$, Silvia Cipriani ${ }^{2}$, Silvia Cipriani, M.M. Mauro ${ }^{1}$ \\ ${ }^{1}$ U.O. Nefrologia e Dialisi, \\ ${ }^{2}$ Servizio di Endoscopia Digestiva ASL Roma H - P.O. Albano Laziale
}

idea che un microorganismo potesse essere coinvolto nella patogenesi della malattia ulcerosa peptica risale al 1893. In tale anno, infatti, Bizzozzero (1) segnalò - per la prima volta - la presenza di spirochete e spirilli nello stomaco di cani. All'inizio del XX secolo batteri simili, spiraliformi, furono rinvenuti in materiali clinici provenienti da gastrectomie di pazienti con adenocarcinoma dello stomaco e malattia ulcerosa peptica. Tali reperti furono semplicemente liquidati come fenomeni di contaminazione post mortem: infatti, il detto «no acid $\rightarrow$ no ulcer» riassumeva il pensiero allora dominante in tema di eziopatogenesi dell'ulcera peptica. Bisognerà attendere il 1983 per avere conferma delle precedenti osservazioni. In uno studio pilota condotto su 20 pazienti affetti da gastrite antrale Warren e Marshall (2) riuscirono a dimostrare la presenza di «bacilli tipicamente ricurvi... assai simili morfologicamente al genere Campylobacter». Nel 1989, infine, tale microorganismo ricevette la sua definitiva consacrazio- ne ad opera dell'International Committee on Systematic Bacteriology che lo ascrisse al nuovo genere Helicobacter per il profilo degli acidi grassi, la presenza di flagelli multipli e la capacità di idrolizzare l'urea.

\section{Identikit di H. pylori}

$H$. pylori rappresenta la specie tipo del nuovo genere Helicobacter, microorganismi ricurvi o spiraliformi Gram negativi e provvisti di flagelli (3). Queste caratteristiche sono comuni alla maggior parte dei batteri intestinali muco-associati. $H$. pylori cresce in atmosfera di $\mathrm{O}_{2}$ ridotto (5$15 \%$ ) con aggiunta di $\mathrm{CO}_{2}$, alla temperatura ideale di $37^{\circ} \mathrm{C}$. Tali batteri sono stati separati da quelli del genere Campylobacter in quanto possiedono flagelli rivestiti, un unico profilo degli acidi grassi, differenti chinoni nella catena respiratoria e sequenze multiple di RNA $16 \mathrm{~S}$. H. pylori vive solitamente nello stomaco e richiede l'enzima ureasi per la colonizzazione dello strato mucoso. Il germe possiede molti fattori di virulenza, alcuni dei quali sono elencati nella Tabella I (4). I suoi flagelli permettono la motilità nel succo e nella mucosa gastrica (5). L'ureasi, idrolizzando l'urea del secreto gastrico, genera abbastanza bicarbonato ed ammoniaca intorno al batterio da consentirgli un sicuro passaggio attraverso la barriera acida dello stomaco ed il suo arrivo nel film protettivo mucoso $(6,7)$. Ivi giunto $H$. pylori aderisce ai fosfolipidi quali fosfatidiletanolamina (8), alle glicoproteine sialilate quale il ganglioside GM3 (9) ed all'antigene B di Lewis presente nei soggetti di gruppo $0(10,11)$. Dopo aver aderito allo strato di muco ed alle mucose, il microorganismo libera delle proteasi solubili e fosfolipasi dannose per l'integrità dello strato stesso e delle cellule sottostanti. Per esempio, l' «umettamento» della mucosa gastrica aumenta in presenza di $H$. pylori (12). Ciò può causare la lisi della componente fosfolipidica.

Uno degli aspetti più interessanti della patogenicità del batterio riguarda la cosiddetta «citotossina 
TABELLA I - FATTORI CHE CONTRIBUISCONO ALLA PATOGENICITÀ E VIRULENZA DI H.P.

\begin{tabular}{|c|c|}
\hline FATTORE & AZIONE \\
\hline Forma spirale & Motilità nello strato mucoso \\
\hline Flagelli & Potenziamento motilità nello strato mucoso \\
\hline $\begin{array}{l}\text { Specificità di adesione a: } \\
\text { fosfatidiletanolamina, ganglioside } \\
\text { GM3, antigene B di Lewis }\end{array}$ & $\begin{array}{l}\text { Colonizzazione selettiva delle cellule epiteliali } \\
\text { gastriche muco-secernenti }\end{array}$ \\
\hline Ureasi & Sopravvivenza nell'ambiente gastrico \\
\hline Catalasi & $\begin{array}{l}\text { Sopravvivenza nella mucosa gastrica, } \\
\text { verosimilmente all'interno di fagovacuoli } \\
\text { (che proteggono il batterio dall' } \mathrm{H}_{2} \mathrm{O}_{2} \text { ) }\end{array}$ \\
\hline Fosfolipasi & $\begin{array}{l}\text { Digestione delle membrane cellulari } \\
\text { dell'epitelio e dello strato mucoso }\end{array}$ \\
\hline Proteasi & $\begin{array}{l}\text { Come le fosfolipasi. Aumentano, inoltre, la } \\
\text { solubilità del muco }\end{array}$ \\
\hline Citotossina vacuolizzante & $\begin{array}{l}\text { Danneggiamento delle cellule epiteliali con } \\
\text { conseguente fuoriuscita di nutrienti dallo } \\
\text { strato sottomucoso }\end{array}$ \\
\hline $\begin{array}{l}\text { Proteine chemiotattiche a basso } \\
\text { peso molecolare }\end{array}$ & $\begin{array}{l}\text { Richiamo di neutrofili e cellule mononucleate } \\
\text { con successivo rilascio di specie reattive } \\
\text { dell'ossigeno e di interleuchine }\end{array}$ \\
\hline Modificata da (4) & \\
\hline
\end{tabular}

vacuolizzante».

Si tratta di una proteina del PM di circa $87 \mathrm{kDa}$, espressa in circa il $65 \%$ dei ceppi di $H$. pylori e responsabile della produzione di vacuoli nelle cellule epiteliali. In quasi tutti i pazienti con ulcera duodenale $H$. pylori-associata sono stati isolati ceppi che producono la citotossina vacuolizzante. Il gene che codifica per la proteina citotossica è chiamato vacA ed è stato clonato da Cover et al (13). Il gene vacA è presente in tutti i ceppi di $H$. pylori, ma solo il $65 \%$ di essi produce la proteina attiva. Una seconda proteina del PM di circa 127 $\mathrm{kDa}$, definita citotossina associata al gene $\mathrm{A}$, o cagA, costituisce un marker dell'effetto tossico vacuolizzante; il gene per la cagA è presente solo in associazione all'effetto citotossico vacA. Anticorpi diretti verso la tossina sono presenti in quasi tutti i pazienti con ulcera duodenale (4).

\section{Helicobacter pylori ed uremia cronica}

Alterazioni del tratto superiore dell'apparato gastroenterico (UGI) si osservano frequentemente nei pazienti emodializzati. Ciononostante la letteratura nefrologica recente è abbastanza avara di dati riguardanti le possibili correlazioni tra uremia cronica ed affezioni dell'UGI, nonché delle eventuali associazioni esistenti, in questi pazienti, tra tali condizioni morbose ed Helicobacler Pylori (H.P.) (14-16). Il presente studio è stato condotto in pazienti sottoposti a trattamento emodialiti- co (RRT) al fine di:

a) valutare la prevalenza di affezioni a carico dell'UGI e di eventuali sintomi ad esse associati;

b) ricercare correlazioni tra patologie dell'UGI ed infezione da H.P.;

c) una possibile applicazione clinica dei risultati ottenuti.

\section{Materiale e metodi}

Sono stati considerati 49 pazienti in trattamento emodialitico periodico (29 maschi, 20 femmine), di età anagrafica media 63.2 \pm 9.9 anni (range $=45$ ) e dialitica media $49.5 \pm 39$ mesi (range=36), con Kt/V e PCR > 1.4 (Tab. II). Dell'intera popolazione è stata eseguita la ricerca degli anticorpi (IgG) anti-HP nel siero con metodo: ELISA (CloneSystem ${ }^{\circledR}$ by IFCI CLONESYSTEM S.p.A ). Di questi, 32 pazienti $(65.3 \%$; 18 maschi, 14 femmine; Tab. III) sono stati, contemporaneamente, sottoposti ad e s of a g ogastroduodenoscopia (EGDS) con prelievi bioptici contestuali (2 campioni dall'antro e 2 campioni dal fondo gastrico). Questi ultimi sono stati inviati per la ricerca istologica di $H P$ mediante colorazione con metodo Giemsa. 17 pazienti (34.7\%; 11 maschi, 6 femmine; Tab. IV) sono stati esclusi dall'indagine strumentale a causa di patologie associate che ne sconsigliavano l'esecuzione (cardiopatia ischemica), di terapie farmacologiche a rischio (anticoagulanti orali o FANS) o perché hanno rifiutato il loro consenso. Nessuna complicazione degna di nota ha fatto seguito all'esame endoscopico. Non vi erano differenze statisticamente significative per età anagrafica e dialitica media nei due gruppi in esame $(61.6 \pm 10.4$ anni vs $66.2 \pm 8.4$ anni; $51.3 \pm 35.0$ mesi vs $46.0 \pm 46.6$ mesi). Abbiamo attentamente valutato, attraverso una accurata indagine anamnestica, la presenza o meno di sintomi a carico dell'UGI, particolarmente nausea, pirosi, sensazione di ripienezza gastrica.

Il gruppo di controllo (v. Tab. V) era costituito da 71 pazienti consecutivi (37 maschi, 34 femmine) di età media 51.0 \pm 5.4 anni (range $=56$ ), non 
TABELLA II - DATI EPIDEMIOLOGI DELLA POPOLAZIONE ARRUOLATA

\begin{tabular}{lccc}
\hline $\begin{array}{l}\text { Kt } / \mathrm{V}>1.4 \\
\mathrm{nPCR}>1.4\end{array}$ & Coorti & Età media \pm DS (anni) & Età dialitica media \pm DS $($ mesi) \\
\hline Maschi & 29 & $64.6 \pm 7.9$ & $51.0 \pm 44.2$ \\
Femmine & 20 & $61.3 \pm 12.2$ & $47.3 \pm 3.1$ \\
Totale & 49 & $63.2 \pm 9.9$ & $59.5 \pm 39$ \\
\hline
\end{tabular}

TABELLA III - DATI EPIDEMIOLOGICI DEI PAZIENTI SOTTOPOSTI AD EGDS

Coorti Età media \pm DS (anni) Età dialitica media \pm DS (mesi)

\begin{tabular}{llcc}
\hline Maschi & 18 & $62.5 \pm 7.3$ & $51.0 \pm 44.0$ \\
Femmine & 14 & $61.6 \pm 13.6$ & $42.5 \pm 33.4$ \\
Totale & 32 & $61.6 \pm 10.4$ & $51.3 \pm 3.5$ \\
\hline
\end{tabular}

TABELLA IV - DATI EPIDEMIOLOGI DEI PAZIENTI NON SOTTOPOSTI AD EGDS

Coorti Età media \pm DS (anni) Età dialitica media \pm DS (mesi)

\begin{tabular}{lccc}
\hline Maschi & 11 & $68.0 \pm 7.9$ & $39.3 \pm 55.2$ \\
Femmine & 6 & $63.0 \pm 8.9$ & $58.3 \pm 24.0$ \\
Totale & 17 & $66.2 \pm 8.4$ & $46.0+46.6$ \\
\hline
\end{tabular}

TABELLA V - GRUPPO DI CONTROLLO*

\begin{tabular}{|c|c|c|c|c|}
\hline & Coorti & Età media \pm DS (anni) & $\begin{array}{c}\text { GIEMSA - positività per } H P \\
\text { Nr** } \\
\% * * *\end{array}$ & $\begin{array}{c}\text { GIEMSA - negatività per } H P \\
\mathrm{Nr}^{* *} \\
\%^{* * *}\end{array}$ \\
\hline Maschi & 37 & $48.4 \pm 17.0$ & $\begin{array}{c}22 \\
59.4\end{array}$ & $\begin{array}{c}15 \\
40.6\end{array}$ \\
\hline Femmine & 34 & $49.3 \pm 16.6$ & $\begin{array}{l}16 \\
47\end{array}$ & $\begin{array}{l}18 \\
53\end{array}$ \\
\hline Totale & 71 & $51.0 \pm 15.4$ & $\begin{array}{c}38 \\
53.5\end{array}$ & $\begin{array}{c}33 \\
46.5\end{array}$ \\
\hline \multicolumn{5}{|c|}{$\begin{array}{l}\text { * Pazienti sintomatici, non uremici } \\
\text { ** Numero assoluto di pazienti } \\
\text { *** Percentuale relativa dei pazienti }\end{array}$} \\
\hline
\end{tabular}

uremici, sintomatici, afferenti al Servizio di Endoscopia Digestiva del nostro P.O.

\section{Risultati}

I risultati ottenuti dal presente studio sono riassunti nella Tabella VI.
In essa sono riportati il numero assoluto e la percentuale relativa di ciascuna delle alterazioni istologiche riscontrate all'EGDS, nonché le correlazioni tra ricerca istologica per $H P$ ed affezioni e tra queste ultime e la sierologia per Helicobacter. Dei pazienti emodializzati sottoposti ad EGDS, 15 (46.8\%) erano positivi alla colorazione GIEMSA, mentre $21(65.6 \%)$ presentavano positività agli $\mathrm{Ab}$ anti-HP. Solo 6 pazienti $(12.2 \%)$ erano sintomatici. La distribuzione e la varia associazione dei sintomi considerati sono illustrati nella Tabella VII. Uno solo tra i pazienti aveva reperti normali alla EGDS, istologia negativa per $H P$ e 
TABELLA VI - RISULTATI

\begin{tabular}{|c|c|c|c|c|c|}
\hline & $\begin{array}{l}\mathrm{N}^{*} \\
\% * *\end{array}$ & $\begin{array}{l}\text { GIEMSA-positività } \\
\text { per } H P\left(\mathrm{~N}^{*} / \%^{* *}\right)\end{array}$ & $\begin{array}{l}\text { GIEMSA-negatività } \\
\text { per } H P\left(\mathrm{~N}^{*} / \%^{* *}\right)\end{array}$ & $\begin{array}{l}\text { Ab- } H P \text { positivi } \\
\left(\mathrm{N}^{*} / \%^{* *}\right)\end{array}$ & $\begin{array}{l}\text { Ab- } H P \text { negativi } \\
\left(\mathrm{N}^{*} / \%^{* *}\right)\end{array}$ \\
\hline Pazienti totali $=49$ & & & & Totale $=49$ & Totale $=49$ \\
\hline \multirow[t]{2}{*}{ Gastriti } & 29 & 13 & 16 & 20 & 9 \\
\hline & 90.6 & 44.8 & 55.2 & 68.9 & 31.1 \\
\hline Ulcera peptica & $\begin{array}{c}7 \\
21.8\end{array}$ & $\begin{array}{c}5 \\
71.4\end{array}$ & $\begin{array}{c}2 \\
28.6\end{array}$ & $\begin{array}{c}5 \\
71.4\end{array}$ & $\begin{array}{c}2 \\
28.6\end{array}$ \\
\hline Metaplasia & $\begin{array}{c}3 \\
9.3\end{array}$ & $\begin{array}{c}2 \\
66.6\end{array}$ & $\begin{array}{c}1 \\
33.3\end{array}$ & $\begin{array}{c}2 \\
66.6\end{array}$ & $\begin{array}{c}1 \\
33.3\end{array}$ \\
\hline Poliposi & $\begin{array}{c}3 \\
9.3\end{array}$ & $\begin{array}{c}2 \\
66.6\end{array}$ & $\begin{array}{c}1 \\
33.3\end{array}$ & $\begin{array}{c}2 \\
66.6\end{array}$ & $\begin{array}{c}1 \\
33.3\end{array}$ \\
\hline Malt-lynphoma & $\begin{array}{c}1 \\
3.1\end{array}$ & $\begin{array}{c}1 \\
100\end{array}$ & - & $\begin{array}{c}1 \\
100\end{array}$ & - \\
\hline Duodeniti & $\begin{array}{c}5 \\
15.6\end{array}$ & $\begin{array}{c}4 \\
80\end{array}$ & $\begin{array}{c}1 \\
20\end{array}$ & $\begin{array}{c}5 \\
100\end{array}$ & - \\
\hline Totale & $\begin{array}{c}31 \\
96.8\end{array}$ & $\begin{array}{c}15 \\
46.8\end{array}$ & $\begin{array}{c}17 \\
53.2\end{array}$ & $\begin{array}{c}21 \\
65.6\end{array}$ & $\begin{array}{c}11 \\
34.4\end{array}$ \\
\hline
\end{tabular}

TABELLA VII - SINTOMI GASTROINTESTINALI DEI PZ IN RRT

\begin{tabular}{lll}
\hline & $\mathrm{N}^{*}$ & $\% * *$ \\
\hline Nausea & $2 / 49$ & 4.1 \\
Pirosi & $1 / 49$ & 2.05 \\
Senso di ripienezza gastrica & $6 / 49$ & 12.2 \\
Totale & $6 / 49$ & 12.2 \\
\hline 'Tre pazienti mostravano più di un sintomo & & \\
* Numero assoluto di pazienti & & \\
$* *$ Percentuale relativa dei pazienti & & \\
\hline
\end{tabular}

negatività sierologica.

Nel gruppo di controllo (v. Tab. V) la positività istologica per $H . P$. era del $53.5 \%$ (38 casi; $\mathrm{p}=N S$ ).

\section{Discussione}

I risultati ottenuti dal presente studio mostrano - nei pazienti in RRT - una prevalenza di affezioni dell'UGI su- periore al $90 \%$ - qualora esse vengano attentamente ricercate tramite esame endoscopico e prelievo bioptico. Tali condizioni morbose sono risultate perlopiù asintomatiche o paucisintomatiche. Per tale motivo, la presenza di sintomi a carico dell'UGI non sembra costituire un indizio affidabile nella diagnostica delle alterazioni dell'apparato gastroenterico. Ciò potrebbe, solo in parte, essere spiegato dalla estrema variabilità ed aspecificità dei sintomi stessi e dalle eventuali terapie farmacologiche assunte (chelanti del fosforo antagonisti dei recettori $\mathrm{H}_{2}$, inibitori della pompa protonica).

E ormai accertato che l'infezione da $H P$ costituisce un fattore importante nel determinismo delle affezioni del1'UGI (14-16): attualmente esso viene considerato il principale agente eziologico per la insorgenza di: gastriti - acute e croniche -, ulcera peptica, adenocarcinoma gastrico e linfoma gastrico non-Hodgkin del tessuto linfoide associato alla mucosa (4) (MALT). Pertanto, tale microorganismo può essere ritenuto responsabile delle lesioni descritte anche nei pazienti con insufficienza renale cronica (IRC), soprattutto in considerazione di condizioni favorenti il suo sviluppo, quali l'ambiente uremico (17-19). A tale proposito i dati riportati in letteratura sono assai 
controversi. Taluni Autori affermano che nei pazienti emodializzati la incidenza di infezione da H.P. è maggiore rispetto ai soggetti normali (2021), mentre altri hanno trovato valori simili $\mathrm{o}$ addirittura più bassi rispetto ai pazienti non uremici $(14,15,22$ 24). Gladziwa et al (16) hanno riscontrato, in uno studio condotto su 164 pazienti con vario grado di IRC, una prevalenza di H.P. variabile dal 34 al 54\% dimostrando, altresì, che gli elevati livelli di urea nel sangue e nel succo gastrico di tali pazienti non rappresenta un fattore di rischio per la infezione da $H$. pylori. Alla medesima conclusione sono giunti Özgür et al (25). In uno studio condotto su 201 pazienti essi hanno dimostrato una prevalenza di infezione da H.P. simile nei soggetti in RRT ed in quelli appartenenti al gruppo di controllo non uremico $(60 \%$ dei casi vs $64 \%$ dei controlli).

I dati emersi dal nostro studio sembrerebbero confermare questa tendenza: $46.8 \%$ di positività istologica per $H P$ nei pazienti in trattamento emodialitico vs $53.5 \%$ nei controlli $(\mathrm{p}=N S)$.

La ricerca degli anticorpi anti- $H P$, pur mostrando una buona correlazione con le alterazioni istologiche, sembra tuttavia sovrastimare la presenza di patologie dell'UGI $H P$-correlate $(65.6 \%$ vs $46.8 \%)$. A tal proposito ci si potrebbe domandare se esiste un gap tra la persistenza dei livelli di anticorpi anti-HP e la «clearance» del microorganismo. Tuttavia, in considerazione della facilità di esecuzione, del basso costo e della scarsa invasività, tale test può essere utilizzato come indagine di primo livello - anche nei pazienti asintomatici - prima di avviarli, eventualmente, a procedure più costose ed invasive.

\section{Conclusioni}

I dati ottenuti mostrano, nei pazienti in RRT, una prevalenza di affezioni dell'UGI $>90 \%$ - qualora esse vengano attentamente ricercate tramite esame endoscopico e prelievo bioptico. Tali pazienti sono perlopiù asintomatici o paucisintomatici. Sebbene l'infezione da $H P$ sia un fattore importante nel determinismo delle lesioni dell'UGI in tutti i pazienti, non abbiamo riscontrato differenze statisticamente significative in quelli uremici rispetto ai controlli non uremici. La ricerca degli anticorpi anti-HP può costituire un utile ausilio nell' $i$ ter diagnostico delle patologie a carico dell'UGI.

\section{BIBLIOGRAFIA}

1. Bizzozzero G. Üeber die schlauchformingen Drusen Des Magendarmkanals und die Beziehungen ihres Epithels zu dem Oberflachenepithel der Schleimhaut. Arch Mikr Anat 1893; 42: 82.

2. Marshall BJ, Warren JR. Unidentified curved bacilli in the stomach of patients with gastritis and peptic ulceration. Lancet i: 1984; 1311.

3. Lee A, ORourke J. Gastric bacteria other than Hellcobacter pylori. Gastroenterol Clin North Am 1993; 22: 21-42.

4. Marshall BJ. Helicobacter pylori. Am J of Gastroenterol 1994; 89: S116.

5. Hazell SL, Lee A, Brady L, et al. Campylobacter pyloridis and gastritis: Association with intercellular spaces and adaptation to an envoronment of mucus as important factors in colonization of the gastric epithelium. J Infect Dis 1986; 153: 658-63.

6. Marshall BJ, Barret LJ, Prakash C, et al. Urea protects Helicobacter (Campylobacter) pylori from the bactericidal effect of acid. Gastroenterology 1990; 99: 697-702.

7. Murakami M, Yoo Jk, Teramura S, et al. Generation of ammonia and mucosal lesion formation following hydrolysis of urea by urease in the rat stomach. J Clin Gastroenterol 1990; 12: s104-9.

8. Gold BD, Huesca M, Sherman PM, et al: Helicobacter mustelae and Helicobacter pylori bind to common lipid receptor in vitro. Infect Immun 1993; 61: 2362-8.

9. Slomiany BL, Pietrowski J, Samanta A, et al. Campylobacter pylori colonization factor shows specificity for lactosylceramide sulfate and GM3 ganglioside. Biochem Intern 1989, 19: 929-36.

10. Dickey W, Collins JS, Watson RG, et al. Secretor status and Heli- 
cobacter pylori infection are independent risk factors for gastroduodenal disease. Gut 1993; 34: 351-3.

11. Boren T, Falk P, Roth KA, et al. Attachment of Helicobacter pylori to human gastric epithelium mediated by blood group antigens. Science 1993; 262 (5141): 1982-5.

12. Goggin PM. Northfield TC, Spychal RT. Factors affecting gastric mucosal hydrophobicity in man. Scand J Gastroenterol 1991, 181 (suppl); 65-73.

13. Tummuru MK, Cover TL, Blaser MJ. Cloning and expression of a high-molecular mass major antigen of Helicobacter pylori: Evidence of linkage to citotoxin production. Infect Immun 1993; 61: 1799-809.

14. Shousha S, Arnout AH, Abbas SH et al. Antral Helicobacter pylori in patients with chronic renal failure. J Clin Pathol 1990; 43: 397.

15. Ali-Kaila K, Vaajalahti P, Karvoran AL, et al. Gastric Helicobacter and upper gastrointestinal symptoms in chronic renal failure. AnnMed 1991; 23: 403.

16. Gladziwa U, Haase G, Handt $\mathrm{S}$, et al. Prevalence of Helicobacter pylori in patients with chronic renal failure. Nephrol Dial Transplant 1993; 8: 301.

17. Lieber CS, Lefèvre A. Ammonia as a source of gastric hypoacidity in patients with uremia. J Clin Invest 1959; 1271-7.

18. Kaess H. Urea in gastric juice. Klin Wochenschr 1970; 48: 124546.

19. Kim H, Park C, Jang WI et al. The gastric juice urea and ammonia levels in patients with Campylobacter pylori. Am J Clin Pathol 1990; 94; 187-91.

20.

Tielemans C, Verhas M, Glupczynski Y, et al. Urea-C 14 breath test screening for Campylobacter pylori infection in uraemic patients. In: Mègraud F, Lamouliatte $\mathrm{H}$, ed Workshop Gastroduodenal Pathology and Campylobacter pylori. Abstract book: 1988: 160.
21. Dervenitios V, Koliouskas D, Kalekou H et al. Campylobacter pylori (C.p.) in severely uremic,hemodialyzed and successfully transplanted patients. Abstact $\mathrm{Xl}^{\text {th }}$ Int Congress of Nephrology, Tokio (Japan): 1990: 138A.

22. Offerhaus GJA, Kreuning J, Velentijn RM et al. Campylobacter pylori: Prevalence and significance in patients with chronic renal failure. Clin Nephrol 1989; 32: 239-41.

23. Conz P, Feriani M, Milan M, Bernardini D, Crepaldi C, La Greca G. Campylobacter pylori infection in uremic dialyzed patients. Nephron 1990; 55: 442-3.

24. Davemport A, Shallcross TM, Crabtree JE, et al. Prevalence of $\mathrm{He}$ licobacter pylori in patients with end-stage renal failure and renal transplant recipients. Nephron 1991; 59: 597601.

25. Özgur O, Boyacioglu S, Özdogan $\mathrm{G}$, et al. Helicobacter pylori infection in haemodialysis patients and renal transplant recipients. Nephrol Dial Transplant 1997; 12 : 289-91. 